Vol. V (2010), No. 5, pp. 919-928

\title{
A Metrics-based Diagnosis Tool for Enhancing Innovation Capabilities in SMEs
}

\author{
J. Sepulveda, J. Gonzalez, M. Camargo, M. Alfaro
}

\author{
Juan Sepulveda, Javier Gonzalez, Miguel Alfaro \\ Department of Industrial Engineering, University of Santiago of Chile \\ 3769 Ecuador Ave. Santiago, Chile. PO Box 10233 \\ Mauricio Camargo \\ Nancy-Universite / ERPI (Equipe de Recherche des Processus Innovatifs) \\ 8, rue Bastien Lepage 54010 Nancy Cedex, France
}

\begin{abstract}
Innovation doubtless represents a main strategic lever for the development of small and medium enterprises (SMEs) in many industrial sectors and it comprises new techniques, new products and new processes, as well as new services which lead to better customer service and revenue. However, the basic question of how well the company is equipped with the necessary practices, methodologies, people, and beliefs, is far from being completely answered yet. In this paper, a metrics-based diagnosis tool for measuring and enhancing the innovation capabilities in SMEs is presented along with a set of preliminary results from case-based studies at the local industry. In this paper we propose a new method by studying the competences of SMEs in concepts tied to innovation and by using a specified framework. As a first step, all of the necessary information by using questionnaires with verbal-scales evaluations is compiled. Second, we use a non-compensatory flow-based sorting method with central profiles to identify the current level and to classify the company into predefined levels. Third, a detailed analysis of the obtained values is performed in order to make a personalized recommendation.
\end{abstract}

Keywords: technological innovation, multicriteria decision making, classification methods.

\section{Introduction}

\subsection{Innovation process}

The innovation literature is a fragmented corpus due to the contribution of many scholars with diverse disciplinary backgrounds that try to adopt different ontological and epistemological positions to investigate and analyze this complex and multimensional phenomena. Thus, a variety of approaches [1], [2] and many different measurement methods [3], [4], [5] can be found. Chiesa et al. in [6] describe process and performance as the two foci of innovation management measures; they overlay core processes with a set of enabling processes, the latter describing the deployment of resources, and the effective use of appropriate systems and tools governed by top management leadership and direction. A close link exists between product and process innovations: the majority of the articles address both type of innovations and only few articles considered only process innovations [7]. We can observe this relationship in the definition made by Cormican et al. [8] , that describe the product innovation as a continuous and cross-functional process involving and integrating a growing number of different competences inside the organization. In the area of the variables related to innovation, we can consider important works on frameworks. Adams et al. [3] in a survey develops a synthesized framework of the innovation management 
process consisting of seven categories: inputs management, knowledge management, innovation strategy, organizational culture and structure, portfolio management, project management and commercialization. Boly [9], based on the literature [7] [8], identified the most used practices by innovative enterprises and he classified them under 13 categories or groups. According to the author, these practices constitute the principal actions performed by the enterprises to define their strategy, to guide and impel the innovation processes and make evolve the organization or its methods of work; they develop these practices completely or partially and in a formal or informal way where the level of use of these practices allows to classify the enterprises according to his innovation potential.

\subsection{Measuring innovation by assessment of practices}

Corona in [10] defined an index of potential innovation (IIP), which is calculated by using multicriteria decision making (MCDM) tools, and uses as criteria the 13 innovation practices defined by Boly [9]. These practices are the concrete actions executed by the enterprises to define their strategy, to guide and to impel the innovation processes and to make evolve the organization or its working methods. The index will allow to obtain a classification according to the attitudes and strategies adopted by these enterprises. Based on [11] we can classify companies as: Proactive, Preactive, Reactive and Passive. On the other hand, Morel et al. [12] propose the use of Choquet's Integral to consider the interaction between the different innovation practices, defining an Aggregated Index of potential innovation (APII). Finally, Assielou in [13], besides of adding two new innovation practices, he makes modifications in the system of treatment and practices.

Current index-based methods are actually sorting procedures and present limitations to correctly classify enterprises in this field. In this paper we propose a new method to make the evaluation of innovation levels in the small and medium enterprises by using a specified framework. As a first step, all the necessary information by using questionnaires with verbal-scales evaluations is collected. Then, a non compensatory flow-based sorting method with limiting profiles is applied. The third step consists of a detailed analysis of the values obtained in each evaluation of an enterprise to perform a personalized recommendation for each company about areas to be improved. An example of its application is given.

\section{Construction of the Gathering Tool and Reference Profiles}

\subsection{Gathering Tool}

Based on the works by Assielou [13], Corona [10] and Camargo [14] we use categories of innovation practices $c_{i}$, each with practices belonging to a similar class, as shown below.

\section{Creation / Concept Generation $\left(c_{1}\right)$}

1.1 Use of tools to increase the creativity

1.2 Integration of the clients and suppliers in the conception process

1.3 Organization, compilation and management of information from the exterior

II. Conception Activities ( $c_{2}$ )

2.1 Use of tools of help to the conception

2.2 Existence of a methodology of help to the conception

2.3 Hardware Equipment

III. Human Resources Management $\left(c_{3}\right)$ 
3.1 Management of competences and the skills of the society

3.2 Innovation stimulation

IV. Strategy $\left(\mathrm{c}_{4}\right)$

4.1 Strategy integrated to favor the innovation

4.2 Network operation

4.3 Client Importance

4.4 Financing

V. Project management $\left(c_{5}\right)$

5.1 Project administration

5.2 Management of project briefcase

5.3 Organization of tasks tied to the Innovation

VI. Capitalization of Ideas and Concepts $\left(c_{6}\right)$

6.1 Continuous Improvement of the innovation process

6.2 Politics of Management of the intellectual property

6.3 Knowledge Capitalization

In order to measure every concept on each category, verbal scales mapped onto five numerical levels are defined with values $\{0 ; 0.25 ; 0.5 ; 0.75 ; 1\}$, where 0 and 1 are the lowest and the highest level, respectively. Each level was defined in detail for each concept, in order to avoid ambiguity and make the evaluation easier to the interviewer; a survey with 18 evaluations grouped into six categories is then applied.

\subsection{Reference Profiles}

By observing the classification established by Godet [11] and the six categories above, it is possible to establish intervals in which we can classify the enterprises; Passive enterprises have the lowest values in every category whereas the Preactive ones obtain the highest values. The division by interval in each category allows us to establish segments of values for each innovation profile in the characteristics to be measured by having into consideration that the values included in the interval belongs to the levels expected for a company of that profile. This can give us the idea that it is possible to determine reference profiles, where for example a Proactive company will have all its evaluation values in the highest intervals. Thus, each of the four divisions represents to a Reference company with Passive, Reactive, Preactive and Proactive characteristics (Table 1). Is it possible to see that these Reference profiles represent companies that have homogeneous development levels in each characteristic, which is not always the case; for instance, there exist companies with high levels of development in Innovation Stimulation, but at the same time poor levels in Knowledge Capitalization, or a company obtains Proactive values in Human Resources Management and Reactive values in Capitalization of Ideas and Concepts. We cannot hope that the majority of the companies will be homogeneous, therefore it is necessary to find out a method that allows us to establish a correct classification within the four profiles using a non compensatory mathematical tool.

\section{FlowSort Method}

Based on the ranking methodology of PROMETHEE, a new sorting method developed by Nemery and Lamboray [15] is proposed for assigning actions to completely ordered categories; 


\begin{tabular}{|c||c|c|c|c|}
\hline Category & Passive & Reactive & Preactive & Proactive \\
\hline \hline I. Creation / Concept Generation & {$\left[0-a_{2}[\right.$} & {$\left[a_{2}-a_{3}[\right.$} & {$\left[a_{3}-a_{4}[\right.$} & {$\left[a_{4}-1\right]$} \\
II. Conception Activities & {$\left[0-b_{2}[\right.$} & {$\left[b_{2}-b_{3}[\right.$} & {$\left[b_{3}-b_{4}[\right.$} & {$\left[b_{4}-1\right]$} \\
III. HR Management & {$\left[0-c_{2}[\right.$} & {$\left[c_{2}-c_{3}[\right.$} & {$\left[c_{3}-c_{4}[\right.$} & {$\left[c_{4}-1\right]$} \\
IV. Strategy & {$\left[0-d_{2}[\right.$} & {$\left[d_{2}-d_{3}[\right.$} & {$\left[d_{3}-d_{4}[\right.$} & {$\left[d_{4}-1\right]$} \\
V. Project management & {$\left[0-e_{2}[\right.$} & {$\left[e_{2}-e_{3}[\right.$} & {$\left[e_{3}-e_{4}[\right.$} & {$\left[e_{4}-1\right]$} \\
VI. Capitalization of Ideas & {$\left[0-f_{2}[\right.$} & {$\left[f_{2}-f_{3}[\right.$} & {$\left[f_{3}-f_{4}[\right.$} & {$\left[f_{4}-1\right]$} \\
\hline
\end{tabular}

Table 1: Interval Distribution of Category values

these categories are defined either by limiting profiles or by central profiles (also named centroids). The assignment of an action into a Category is based on the relative position of this action with respect to the defined reference profiles in terms or incoming or outgoing net flows. We denote by $A:\left(a_{1}, \ldots, a_{n}\right)$ the set of $n$ actions to be sorted. These actions are evaluated on $q$ criteria $g_{j}(j=1, \ldots, q)$ that have to be maximized. We denote the categories to which the actions must be assigned by $C_{1}, C_{2}, \ldots, C_{k}$. These categories are either delimited by two boundaries, in the case of limiting profiles, or by centroids in the case of central profiles. This Categories are ordered as $C_{1}>\ldots C_{l}>C_{k}$, where $C_{h}>C_{k}$, with $h<l$, which denotes that $C_{h}$ is preferred to category $C_{l}$. We denote $R=\left(r_{1} \ldots, r_{k+1}\right)$ as the set of limiting profiles in the case when a category is defined by an upper and lower profile, represented as $r_{h+1}$. On the other hand, when we define a category by one central profile, the centroid is denoted by $\tilde{R}=\left(\tilde{r}_{1}, \ldots, \tilde{r}_{k}\right)$, where $\tilde{r}_{j}$ is the centroid of category $C_{j}$. We also define $\pi(x, y)$ as the preference of action $x$ over an action $y$, which is used in the same way as in PROMETHEE. Thus, on the basis of these preference degree, positive, negative and net flows of each action $x$ of $R_{i}$, are computed by equations $(3.1),(3.2),(3.3)$, where $R_{i}=R \bigcup\left\{a_{i}\right\}$.

$$
\begin{gathered}
\phi_{\dot{\mathrm{R}}_{i}}^{+}=\frac{1}{\left|\dot{\mathrm{R}}_{i}\right|-1} \sum_{y \in \dot{\mathrm{R}}_{i}} \pi(x, y) \\
\phi_{\dot{\mathrm{R}}_{\mathrm{i}}}^{-}=\frac{1}{\left|\dot{\mathrm{R}}_{i}\right|-1} \sum_{y \in \dot{\mathrm{R}}_{i}} \pi(y, x) \\
\phi_{\dot{\mathrm{R}}_{i}}=\phi_{\dot{\mathrm{R}}_{i}}^{+}-\phi_{\dot{\mathrm{R}}_{i}}^{-}
\end{gathered}
$$

In this case we use $\dot{R_{i}}$ when no difference can be made between a set of limiting profiles and a set of centroids.

The Flow-Based Assignment rules differ in the use of limiting profiles and central profiles. In the case of limiting profiles, the rules of the positive and negative flow assignment are defined as follows:

$$
\begin{aligned}
& C_{\phi^{+}}\left(a_{i}\right)=C_{h}, \text { if } \phi_{R_{i}}^{+}\left(r_{h}\right) \geq \phi_{R_{i}}^{+}\left(a_{1}\right)>\phi_{R_{i}}^{+}\left(r_{h+1}\right) \\
& C_{\phi^{-}}\left(a_{i}\right)=C_{h}, \text { if } \phi_{R_{i}}^{-}\left(r_{h}\right)<\phi_{R_{i}}^{-}\left(a_{1}\right) \leq \phi_{R_{i}}^{-}\left(r_{h+1}\right)
\end{aligned}
$$

If we want to strictly impose the assignment to one category, using the net flow we can define the assignment rule by (3.6).

$$
C_{\phi}\left(a_{i}\right)=C_{h}, \text { if } \phi_{R_{i}}\left(r_{h}\right) \geq \phi_{R_{i}}\left(a_{1}\right)>\phi_{R_{i}}\left(r_{h+1}\right)
$$


In the case of central profiles, the Flow-Based Assignment rules of positive and negative flows are defined by (3.7) and (3.8).

$$
\begin{aligned}
& \tilde{C}_{\phi^{+}}\left(a_{i}\right)=C_{h} \text {, if } \frac{\phi_{\tilde{R}_{i}}^{+}\left(\tilde{r_{h}}\right)+\phi_{\tilde{R}_{i}}^{+}\left(\tilde{r}_{h+1}\right)}{2}<\phi_{\tilde{R}_{i}}^{+}\left(a_{1}\right) \leq \frac{\phi_{\tilde{R}_{i}}^{+}\left(\tilde{r_{h}}\right)+\phi_{\tilde{R}_{i}}^{+}\left(\tilde{r}_{h-1}\right)}{2} \\
& \tilde{C}_{\phi^{-}}\left(a_{i}\right)=C_{h} \text {, if } \frac{\phi_{\tilde{R}_{i}}^{-}\left(\tilde{r_{h}}\right)+\phi_{\tilde{R}_{i}}^{-}\left(\tilde{r}_{h+1}\right)}{2} \geq \phi_{\tilde{R}_{i}}^{-}\left(a_{1}\right)>\frac{\phi_{\tilde{R}_{i}}^{-}\left(\tilde{r_{h}}\right)+\phi_{\tilde{R}_{i}}^{-}\left(\tilde{r}_{h-1}\right)}{2}
\end{aligned}
$$

Here, we can also strictly impose the assignment to one category using the net flows with the assignment rule (3.9).

$$
\tilde{C}_{\phi}\left(a_{i}\right)=C_{h}, \text { if } \frac{\phi_{\tilde{R}_{i}}\left(\tilde{r_{h}}\right)+\phi_{\tilde{R}_{i}}\left(\tilde{r}_{h+1}\right)}{2}<\phi_{\tilde{R}_{i}}\left(a_{1}\right) \leq \frac{\phi_{\tilde{R}_{i}}\left(\tilde{r}_{h}\right)+\phi_{\tilde{R}_{i}}\left(\tilde{r}_{h-1}\right)}{2}
$$

\section{Application}

In this section the method is explained in four main steps, by using information obtained from seven SMEs, $E_{j}$, with $(j=1, \ldots, 7)$ taken from the metalworking industry located at Santiago of Chile, as follows.

$E_{1}$ : appliances manufacturing such as refrigerators, gas and kerosene heaters.

$E_{2}$ : appliances manufacturing such as gas and electric stoves and heaters.

$E_{3}$ : appliances manufacturing such as home boilers and sinks.

$\mathrm{E}_{4}$ : faucets and gas valves manufacturing.

$E_{5}$ : vehicle transforming maker such as for ambulances and safety vehicles.

$E_{6}$ : safety deposit box manufacturing with electronic controls.

$E_{7}$ : vending machine refurbishment and adapting for industrial utilization.

\subsection{First Step: Determination of the weights and references profiles.}

By observing the weight used in each one of the practices in [13], we construct our own weight distribution, considering that our definition of categories and concepts is a grouping and in some cases the division of the innovation practices. This weight distribution can be observed in Table 2 , where also for every category the local weight of each concept of a category is indicated.

To establish the reference profiles, we will define four areas for each of the six categories using the construction of limiting profiles; this will allow us to establish min and max values in every category $c_{i}$. In this case we constructed a symmetrical division in each of the categories because the necessary information to establish central profiles was not available (Table 3).

\subsection{Second step: Survey application and data processing.}

In this step we work with the information collected from the surveys made to each enterprise. According to the evaluation of each of the concepts, an evaluation for each category $c_{i}$ is made by the weighted sum $\sum e_{i j} w_{i j}$, where $e_{i j}$ is the evaluation between $[0,1]$ of the $j$-th concept in the $i$-th category, and $w_{i j}$ is the local weight (Table 4 ). 


\begin{tabular}{|c|c|c|}
\hline Category $\left(c_{i}\right)$ & Local Concept Weights $\left(w_{i j}\right)$ & Global Category Weight $\left(w_{i}\right)$ \\
\hline \hline I. Creation / Concept Generation & $\{0.26 ; 0.33 ; 0.41\}$ & 0.175 \\
II. Conception Activities & $\{0.43 ; 0.19 ; 0.38\}$ & 0.107 \\
III. Human Resources Management & $\{0.47 ; 0.53\}$ & 0.068 \\
IV. Strategy & $\{0.05 ; 0.51 ; 0.27 ; 0.17\}$ & 0.232 \\
V. Project management & $\{0.01 ; 0.47 ; 0.52\}$ & 0.194 \\
VI. Capitalization of Ideas and Concepts & $\{0.43 ; 0.29 ; 0.37\}$ & 0.224 \\
\hline
\end{tabular}

Table 2: Local and Global weights concepts

\begin{tabular}{|c||c|c|c|c|c|c|}
\hline Limiting Profile & $\mathrm{c}_{1}$ & $\mathrm{c}_{2}$ & $\mathrm{c}_{3}$ & $\mathrm{c}_{4}$ & $\mathrm{c}_{5}$ & $\mathrm{c}_{6}$ \\
\hline \hline $\mathrm{r}_{1}$ & 1 & 1 & 1 & 1 & 1 & 1 \\
$\mathrm{r}_{2}$ & 0.75 & 0.75 & 0.75 & 0.75 & 0.75 & 0.75 \\
$\mathrm{r}_{3}$ & 0.5 & 0.5 & 0.5 & 0.5 & 0.5 & 0.5 \\
$\mathrm{r}_{4}$ & 0.25 & 0.25 & 0.25 & 0.25 & 0.25 & 0.25 \\
$\mathrm{r}_{5}$ & 0 & 0 & 0 & 0 & 0 & 0 \\
\hline
\end{tabular}

Table 3: Limiting Profiles defined in Flow-Sort Method

\begin{tabular}{|c||c|c|c|c|c|c|}
\hline $\mathrm{A}$ & $\mathrm{c}_{1}$ & $\mathrm{c}_{2}$ & $\mathrm{c}_{3}$ & $\mathrm{c}_{4}$ & $\mathrm{c}_{5}$ & $\mathrm{c}_{6}$ \\
\hline \hline $\mathrm{E}_{1}$ & 0.31 & 0.32 & 0.12 & 0.13 & 0 & 0 \\
$\mathrm{E}_{2}$ & 0.36 & 0.59 & 0.40 & 0.86 & 0.15 & 0.19 \\
$\mathrm{E}_{3}$ & 0.28 & 0.16 & 0.40 & 0.58 & 0 & 0.10 \\
$\mathrm{E}_{4}$ & 0.17 & 0.22 & 0.12 & 0.43 & 0.11 & 0.19 \\
$\mathrm{E}_{5}$ & 0.26 & 0.42 & 0.24 & 0.34 & 0.15 & 0 \\
$\mathrm{E}_{6}$ & 0.52 & 0.78 & 0.52 & 0.98 & 0.64 & 0.42 \\
$\mathrm{E}_{7}$ & 0.17 & 0.2 & 0.18 & 0.29 & 0.34 & 0.11 \\
\hline
\end{tabular}

Table 4: Evaluation results

\subsection{Third Step: Flow-Sort Aplication}

By defining the set of actions for the seven enterprises as $A=\left\{E_{1}, E_{2}, E_{3}, E_{4}, E_{5}, E_{6}, E_{7}\right\}$, which have been evaluated in the six criteria already defined, and the four classification categories \{Passive, Reactive, Preactive, Proactive $\}$ defined by the five limiting profiles of Table 3, we start calculating the preference degrees, showed in Table 5 between the reference profile and the seven enterprises in order to obtain positive and negative flows. With these calculations we can measure positive, negative, and net flows for each enterprise by using equations ( 3.1$)$, ( 3.2 ), and (3.3). The calculations of all of the flows for the enterprises is shown in Table 6, where for example the positive flow of enterprise 1 with respect to limiting profile $r_{4}$ is calculated as

$$
\phi_{R_{1}}^{+}\left(r_{4}\right)=\frac{\sum \pi\left(r_{4}, r_{j}\right)+\pi\left(r_{4}, E_{1}\right)}{\left|R_{4}\right|-1}=\frac{1+0.67}{6-1}=0.334
$$

The assignment to each category in Table 6 were obtained by equations ( 3.4 ) and (3.5); for example for assigning Enterprise 1 the flow is between profile limits $r_{4}$ and $r_{5}$ as indicated by $\phi_{R_{1}}^{+}\left(r_{4}\right) \geq \phi_{R_{1}}^{+}\left(E_{1}\right) \geq \phi_{R_{1}}^{+}\left(r_{5}\right)$ and $\phi_{R_{1}}^{-}\left(r_{4}\right)<\phi_{R_{1}}^{-}\left(E_{1}\right) \leq \phi_{R_{1}}^{-}\left(r_{5}\right)$. Thus Enterprise 1 is classified as Passive. In the cases when positive and negative flows difers, for example Enterprise 3 and 5 , we must apply the equation (3.6) to obtain an unique classification. 


\begin{tabular}{|c||c|c|c|c|c|}
\hline & $r_{1}$ & $r_{2}$ & $r_{3}$ & $r_{4}$ & $r_{5}$ \\
\hline$\pi\left(E_{1}, r_{j}\right)$ & 0 & 0 & 0 & 0.33 & 0.67 \\
$\pi\left(r_{j}, E_{1}\right)$ & 1 & 1 & 1 & 0.67 & 0 \\
\hline$\pi\left(E_{2}, r_{j}\right)$ & 0 & 0.17 & 0.33 & 0.67 & 1 \\
$\pi\left(r_{j}, E_{2}\right)$ & 1 & 0.83 & 0.67 & 0.33 & 0 \\
\hline$\pi\left(E_{3}, r_{j}\right)$ & 0 & 0 & 0.17 & 0.50 & 0.83 \\
$\pi\left(r_{j}, E_{3}\right)$ & 1 & 1 & 0.83 & 0.50 & 0 \\
\hline$\pi\left(E_{4}, r_{j}\right)$ & 0 & 0 & 0 & 0 & 1 \\
$\pi\left(r_{j}, E_{4}\right)$ & 1 & 1 & 1 & 1 & 0 \\
\hline$\pi\left(E_{5}, r_{j}\right)$ & 0 & 0 & 0 & 0.50 & 0.83 \\
$\pi\left(r_{j}, E_{5}\right)$ & 1 & 1 & 0 & 0.50 & 0 \\
\hline$\pi\left(E_{6}, r_{j}\right)$ & 0 & 0 & 0 & 0.50 & 0.83 \\
$\pi\left(r_{j}, E_{6}\right)$ & 1 & 1 & 1 & 0.50 & 0 \\
\hline$\pi\left(E_{7}, r_{j}\right)$ & 0 & 0 & 0 & 0.33 & 1 \\
$\pi\left(r_{j}, E_{7}\right)$ & 0 & 0 & 0 & 0.67 & 0 \\
\hline
\end{tabular}

Table 5: Preference degrees between the reference profile and the actions

\begin{tabular}{|c||c|c|c|c|c|c|c||c|}
\hline & & $\mathrm{r}_{1}$ & $\mathrm{r}_{2}$ & $\mathrm{r}_{3}$ & $\mathrm{r}_{4}$ & $\mathrm{r}_{5}$ & $\mathrm{E}_{\mathrm{i}}$ & Class \\
\hline \hline \multirow{3}{*}{$\mathrm{R}_{1}$} & $\phi_{+}$ & 1 & 0.8 & 0.6 & 0.344 & 0 & 0.173 & Passive \\
& $\phi_{-}$ & 0 & 0.2 & 0.4 & 0.656 & 0.916 & 0.744 & Passive \\
& $\phi_{\text {net }}$ & 1 & 0.6 & 0.2 & -0.313 & -0.916 & -0.571 & Passive \\
\hline \multirow{3}{*}{$\mathrm{R}_{2}$} & $\phi_{+}$ & 1 & 0.754 & 0.532 & 0.284 & 0.0 & 0.431 & Reactive \\
& $\phi_{-}$ & 0 & 0.246 & 0.468 & 0.716 & 1 & 0.569 & Reactive \\
& $\phi_{\text {net }}$ & 1 & 0.507 & 0.064 & -0.433 & -1 & -0.139 & Reactive \\
\hline \multirow{3}{*}{$\mathrm{R}_{3}$} & $\phi_{+}$ & 1 & 0.8 & 0.554 & 0.305 & 0 & 0.303 & Passive \\
& $\phi_{-}$ & 0 & 0.2 & 0.446 & 0.695 & 0.961 & 0.659 & Reactive \\
& $\phi_{\text {net }}$ & 1 & 0.6 & 0.107 & -0.39 & -0.961 & -0.356 & Reactive \\
\hline \multirow{3}{*}{$\mathrm{R}_{4}$} & $\phi_{+}$ & 1 & 0.8 & 0.6 & 0.354 & 0 & 0.246 & Passive \\
& $\phi_{-}$ & 0 & 0.2 & 0.4 & 0.646 & 1 & 0.754 & Passive \\
& $\phi_{\text {net }}$ & 1 & 0.6 & 0.2 & -0.293 & -1 & -0.507 & Passive \\
\hline \multirow{3}{*}{$\mathrm{R}_{5}$} & $\phi_{+}$ & 1 & 0.8 & 0.6 & 0.297 & 0 & 0.258 & Passive \\
& $\phi_{-}$ & 0 & 0.2 & 0.4 & 0.703 & 0.955 & 0.697 & Reactive \\
& $\phi_{\text {net }}$ & 1.0 & 0.6 & 0.2 & -0.406 & -0.955 & -0.439 & Passive \\
\hline \multirow{3}{*}{$\mathrm{R}_{6}$} & $\phi_{+}$ & 1.0 & 0.732 & 0.445 & 0.2 & 0 & 0.623 & Preactive \\
& $\phi_{-}$ & 0.0 & 0.268 & 0.555 & 0.8 & 1 & 0.377 & Preactive \\
& $\phi_{\text {net }}$ & 1.0 & 0.464 & -0.11 & -0.6 & -1 & 0.246 & Preactive \\
\hline \multirow{3}{*}{$\mathrm{R}_{7}$} & $\phi_{+}$ & 1.0 & 0.8 & 0.6 & 0.315 & 0 & 0.285 & Passive \\
& $\phi_{-}$ & 0.0 & 0.2 & 0.4 & 0.685 & 1 & 0.715 & Passive \\
& $\phi_{\text {net }}$ & 1.0 & 0.6 & 0.2 & -0.37 & -1 & -0.43 & Passive \\
\hline \multirow{2}{*}{} & & & & & & & & \\
& & & & & &
\end{tabular}

Table 6: Flow-Sort Results

The assignment of an enterprise into one of the four enterprises profiles can be easily displayed using the positive and negative flows diagram. In Figure 1, which shows the flows of the Enterprise 1 , it is possible to view that there is not ambiguity in the classification of this enterprise into the Passive profile, since the positive and negative flows allocate this enterprise into the same profile. In other case, in the Figure 2 which shows the flows of the Enterprise 3, we can observe that the positive flow classifies the enterprise into the Passive profile and the negative flow classifies the enterprise into the Reactive profile, but the final net flow classify this enterprise into the Reactive profile. This final classification can be explained taking into consideration the 
proximity of the positive and negative action score to the Reactive area classification over the Passive area classification.

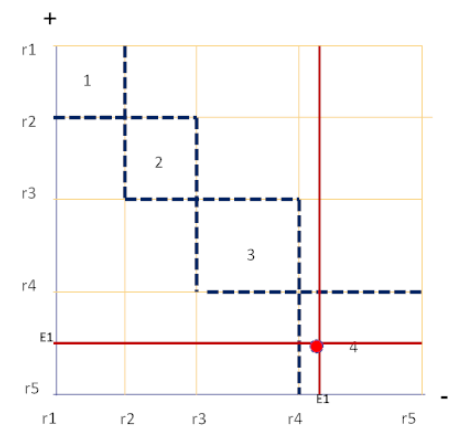

Figure 1: Flow Diagram Enterprise 1

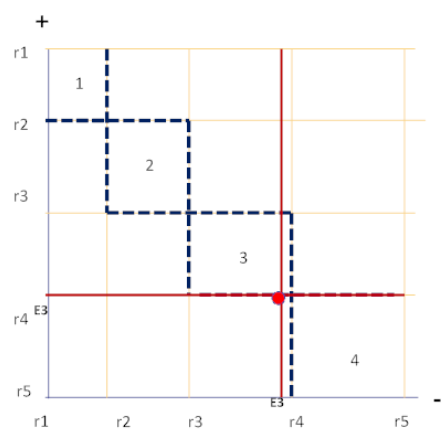

Figure 2: Flow Diagram Enterprise 3

For the Enterprise 6 identified as Preactive in this method, the net flow action obtains a positive value, which is opposed to the net values obtained by the enterprises classified as Passive which have the most negative value (Figure 2). It is important to note that the profile areas where there is not exist any ambiguity depends only on the singular comparison between an enterprise and all of the reference profiles. The former explains the variety of these areas that we see in all these flows diagrams.

\subsection{Fourth Step:Analysis of the results.}

The analysis of the results and the search of possible alternatives of innovation progress in these enterprises can be analyzed by observing the detailed net score flows for each category, which show the net significance of all six Categories in obtaining the final net flow value for every enterprise. We can observe that in most cases the category that contributes with the highest negative flows is the Category Capitalization of Ideas, which is responsible for the third part of the total net flow. The second category with the most negative flows is the category Project Management. In most enterprises, these two categories grouped together represent between the $40 \%$ or $50 \%$ of the final significance in the net flow action (Table 7). This can give us an idea

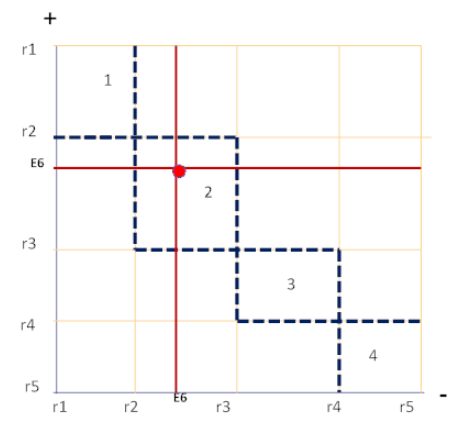

Figure 3: Flow Diagram Enterprise 6 
that any improvement in the values obtained in the values of these categories can be critical to obtain a better evaluation. In other words, any improvement in any of the concepts that belongs to the categories above mentioned may produce an advance of the innovation process. Thus, these enterprises would be more qualified to move to a higher profile. On the other hand, in the enterprises that obtained a classification as Reactive and Preactive, the values associated to the Category Strategy have positive net flows values having a positive significance into the total net flow action; thus we can say that enterprises with good evaluations in the concepts concerning to the Strategy can present features of a Reactive or a Preactive enterprise profile.

\begin{tabular}{|c||c|c|c|c|c|c|}
\hline & $\mathrm{c}_{1}$ & $\mathrm{c}_{2}$ & $\mathrm{c}_{3}$ & $\mathrm{c}_{4}$ & $\mathrm{c}_{5}$ & $\mathrm{c}_{6}$ \\
\hline \hline $\mathrm{E}_{1}$ & $6.13 \%$ & $3.75 \%$ & $7.15 \%$ & $24.39 \%$ & $27.19 \%$ & $31.39 \%$ \\
$\mathrm{E}_{2}$ & $7.61 \%$ & $4.65 \%$ & $2.96 \%$ & $30.26 \%$ & $25.30 \%$ & $29.22 \%$ \\
$\mathrm{E}_{3}$ & $7.80 \%$ & $14.30 \%$ & $3.03 \%$ & $10.34 \%$ & $34.58 \%$ & $29.95 \%$ \\
$\mathrm{E}_{4}$ & $20.70 \%$ & $12.66 \%$ & $8.04 \%$ & $9.15 \%$ & $22.95 \%$ & $26.50 \%$ \\
$\mathrm{E}_{5}$ & $7.97 \%$ & $4.87 \%$ & $9.29 \%$ & $10.56 \%$ & $26.50 \%$ & $40.80 \%$ \\
$\mathrm{E}_{6}$ & $10.43 \%$ & $19.13 \%$ & $4.05 \%$ & $41.48 \%$ & $11.56 \%$ & $13.35 \%$ \\
$\mathrm{E}_{7}$ & $24.44 \%$ & $14.94 \%$ & $9.50 \%$ & $10.80 \%$ & $9.03 \%$ & $31.28 \%$ \\
\hline
\end{tabular}

Table 7: Detailed Net Flow Significance

\section{Conclusion}

In this paper a new enterprise classification method by using a non compensatory flowbased sorting method with limiting profiles has been proposed. The method called Flow-Sort was used to identify the current level of the enterprise and to classify it into four predefined levels of innovation: Passive, Reactive, Preactive, and Proactive. The aim of the method, besides classifying the enterprise into a profile, is to give new ideas to formulate an improvement strategy to allow the company to increase its innovation performance. Along this work, we established many observations, as $(a)$ the importance of defining a precise framework that allow us to evaluate all the characteristics and concepts in a precise and structured form, $(b)$ the correct construction of the tool for gathering data, since this is a key element for obtaining the necessary information input, $(c)$ the use of a mathematical tool that allows a comparison against an established profile; the tool is independent of the universe of enterprises to be measured so that it can be applied on a reduced or a large number of enterprises without changing its effectiveness, (d) the possibility of establishing the parameters for an innovation improvement strategy for each of the enterprises individually according to their obtained values and the analysis of significance of each of the categories and concepts.

As a limitation of the application used in the example above, we have the highly arbitrary definition of the four reference profiles since we used four homogenous zones for the six categories when using limiting profiles, as shown in Table 3. More accurate knowledge on a company may allow different values for the profiles, or better the use of central profiles. The latter may be continuously refined as the analysis is repeated in the mid and long term. A classification into new innovation levels can be made by using the proposed method. In such a case, the use of either central or limiting profiles will exclusively depend on the certainty about the characteristics of each new level.

Another comment is related to the interpretation of results and the creation of incentive policies towards better innovation performance levels; these aspects are left to the policy makers at each company since they cannot be predefined in a standardized way. However, an empirical study 
with a broader universe of companies by using the method here proposed could give a better answer on the suitability of best practices of this field.

\section{Bibliography}

[1] Kerssens-van Drongelen, I.C. and Bilderbeek, J., R\&D performance measurement: more than choosing a set of metrics, R\& D Management vol 29, nş1, p. 35-46, 1999

[2] Koberg C., Detienne D., Heppard k.,An empirical test of environmental, organizational, and process factors affecting incremental and radical innovation, Journal of High Technology Management Research 14 p 21-45, 2002

[3] Adams, R., Bessant, J., Phelps, R., Innovation management measurement: A review, International Journal of Management Reviews, vol 8, nş1, p. 21-47. 2006

[4] Bremser W., Barsky N., Utilizing the balanced scorecard for R\&D 5 performance measurement, Management vol 34 nş3, p. 229-238, 2004

[5] Wang C.,Lu I., Chen I, Evaluating firm technological innovation 15 capability under uncertainty, Technovation vol 28, p. 349-, 2008

[6] Chiesa, V., Coughlan, P., Voss, C.A., Development of a technical innovation audit, Journal of Product Innovation Management 13 (2), p 105-136, 1996

[7] Becheikh N., Landry, R., Amara, N., Lessons from innovation empirical studies in the manufacturing sector: A systematic review of the literature from 1993-2003, Technovation, vol 26, nş5-6, p. 644-664,2005

[8] Cormican k.,Sullivan D.,Auditing best practice for effective product innovation management, Technovation vol 24, p. 819-829, 2004

[9] Boly, V., Ingénierie de l'innovation : organisation et methodologies des entreprises innovantes,Lavoisier, Paris, France, 2004

[10] Corona A. José Ramón, Innovation et metrologie : une approche en terme d'Indice d'Innovation Potentielle, Thčse de Doctorat, Institut Nationale Polytechnique de Lorraine, février, 2005

[11] Godet, M., Manuel de prospective stratégique. Tome 2. L'art et la methode, Ed. Dunod, Paris, France, 1997

[12] Morel L., Camargo M.,Comparison of multicriteria analysis techniques to improve the innovation process measurement, IAMOT 2006, Beijing, China, 8 pages, may 22-26

[13] Assielou G, Metrologie des processus d'innovation, thčse de Doctorat, Institut Nationale Polytechnique de Lorraine, 2008

[14] Camargo M., Morel L., Fonteix C., Evolutionary based methodology to integrate product innovation degree on a firm technological strategy, IAMOT 2007 Proceedings

[15] Nemery P, Lamboray C. FlowSort: a flow-based sorting method with limiting or central profiles, TOP 16:90-113, Springer-Verlag, 2008 